\title{
Enhancing Dual-task Learning through Supraposture-first Instruction in Healthy Young Adults
}

\author{
Shu-Han $\mathrm{Yu}^{1}$ and Cheng-Ya Huang ${ }^{1,2^{\star}}$ \\ ${ }^{1}$ School and Graduate Institute of Physical Therapy, College of Medicine, National Taiwan University, Taipei, Taiwan \\ ${ }^{2}$ Physical Therapy Center, National Taiwan University Hospital, Taipei, Taiwan
}

\begin{abstract}
Background: Previous studies have demonstrated that the performance of a postural-suprapostural dual task can be modulated by varied attention prioritization. The purpose of this study was to compare the effects of two task-priority approaches to dual-task learning on accuracy and dynamic characteristics of postural-suprapostural performance.

Methods: We concurrently conducted a force-matching precision grip task (suprapostural task) while maintaining a stabilometer stance (postural task). Twenty adults were randomly assigned to one of two learning conditions: (1) dual-task learning with prioritizing force-matching precision grip task (supraposture first, SF), or (2) dual-task learning with prioritizing stabilometer movement (posture first, PF). Force-matching error, postural error, and dynamics of force-matching peak and stabilometer movement were evaluated.

Results: Dual-task learning with the SF strategy caused superior force-matching, postural accuracy, and more complex stabilometer movements than dual-task learning with the PF strategy.

Conclusion: Dual-task learning with the SF strategy takes advantage of more autonomous and flexible postural responses to facilitate the suprapostural performance.
\end{abstract}

\section{Introduction}

Dual-task paradigms have been widely used to evaluate the extent of information-processing sharing requirement of postural tasks with other concurrent tasks. Traditionally, upright posture control is assumed to be an automatic task without higher-level cortical processing, as large-scale stance synergies are well established. In daily life, many activities involve maintaining balance while performing at least one other concurrent task (e.g. walking and texting). The task which is superordinate to the postural control is the suprapostural task [1]. The influence of adding a suprapostural task on balance control has received considerable attention. Growing literature on the performance of postural control functions has shown that maintaining upright balance is a complex physical task, especially while carrying out a suprapostural task $[2,3]$. Task prioritization is often manipulated to better understand the role of attention in postural-suprapostural control. The simultaneous performance of a postural task and a suprapostural task may create a conflict, causing the need to determine which of the tasks receives higher priority, especially when attentional resources to execute both task concurrently are limited [4]. However, much of the literature on dual-tasking has participants performing a postural task and a suprapostural task simultaneously without specific instructions about task prioritization.

While performing a dual-task, the tasks must be appropriately prioritized to achieve goals while maintaining safety. The "posturefirst" concept, originally introduced by Shumway-Cook et al. in 1997, is considered a safe strategy, favoring balance maintenance over execution of a suprapostural task. Some studies reported that healthy young adults are more inclined to use this posture first strategy to prevent fall $[5,6]$. Bloem et al. $(2001,2006)$ even proposed that Parkinson patients who easily fall because they prioritize a "posturesecond" (or "supraposture-first") strategy in daily life $[7,8]$. However, some other dual-task studies reported that healthy young adults might better perform by using the supraposture-first strategy instead of posture first strategy. Healthy young adults were asked to walk on a narrow-base while executing an auditory Stroop test [9]. Focusing on the Stroop test caused a faster response to the Stroop test and did not decrease walking speed. However, when the subjects focused on the walking task, their response time and accuracy for the Stroop test deteriorated significantly. Similarly, while standing on a platform with feet together and performing a visual spatial memory task, focusing on the memory task caused a shorter response time and did not increase postural sway relative to focusing on the postural balance [10]. This phenomenon implies that posture-first is not an invariant strategy and that attention prioritization is flexible, depending on various individual tasks, and environmental factors $[9,11]$.

Few studies have addressed the impact of dual-task learning with variable attention prioritization. The benefit of dual-task learning with switching attention between postural and suprapostural tasks was shown by Silsupadol et al. [12,13] with the variable-priority strategy and participants were asked to shift attention between postural and suprapostural tasks by focusing on balance activities (posture-first) in half the learning session and focusing on cognitive tasks (supraposture-first) in the other half. Both the learning effect and the transfer effect had better outcomes relative to when the participants placed the same amount of attention on balance activities and cognitive tasks (equal priority) during the whole training session. However, to our knowledge, no research has been done examining the effects of various attention prioritization (posture-first vs. supraposture-first) on dual-task learning. By adopting posture-first and supraposture-first strategies for dual-task learning, the main purpose of the present study was to assess which attention prioritization

"Corresponding Author: Dr. Cheng-Ya Huang, School and Graduate Institute of Physical Therapy, College of Medicine, National Taiwan University, Taipei, 100, Taiwan, Tel: 886-2-33668131; E-mail: rcyhuangg@ntu.edu.tw

Citation: Yu SH, Huang CY (2015) Enhancing Dual-task Learning through Supraposture-first Instruction in Healthy Young Adults. Int J Phys Ther Rehab 1 : 105. doi: http://dx.doi.org/10.15344/2455-7498/2015/105

Copyright: @ 2015 Yu et al. This is an open-access article distributed under the terms of the Creative Commons Attribution License, which permits unrestricted use, distribution, and reproduction in any medium, provided the original author and source are credited. 
Citation: Yu SH, Huang CY (2015) Enhancing Dual-task Learning through Supraposture-first Instruction in Healthy Young Adults. Int J Phys Ther Rehab 1: 105. doi: http://dx.doi.org/10.15344/ijptr/2015/105

Page 2 of 5

strategy (posture-first or supraposture-first) optimizes posturalsuprapostural dual-tasking learning. We hypothesized that dual-task learning would change postural and suprapostural performance in relation to the attention prioritization of the dual-task condition.

\section{Method}

\section{Subjects}

The study was conducted with 20 healthy right-handed participants (8 male, 12 female; mean age: $24.5 \pm 3.3$ years) from the university campus. All participants signed an informed consent for the experimental procedure, approved by the local institutional review board (National Taiwan University Hospital Clinical Trail Center) to protect the rights of the subjects. Participants who were not able to maintain their balance on a stabilometer (58-cm length $\times 50$ $\mathrm{cm}$ width $\times 26-\mathrm{cm}$ height) for at least 80 seconds, had history of a neuromuscular system disease, or any injury that could affect their balance, were excluded from this study.

\section{Experimental procedures and system-setup}

The participants were asked to perform a force-matching precision grip task with their right index and thumb while standing on a stabilometer in the postural-suprapostural dual-task condition (Figure 1). For the postural task, participants were asked to maintain seconds for the first three warning-executive pairs, but was randomly presented at different intervals of 1.5, 1.8, 2.1, 2.4, 2.7 or 3.0 seconds from the fourth to fifteenth warning-executive pairs. The interval between the executive tone and the next warning tone was 3.5 seconds. Participants performed a quick thumb-index precision grip (force impulse duration $<0.5$ second) to couple the peak precision force with the force target when receiving the executive tone.

Participants were randomly assigned to either the posture-first group (PF group; 10 subjects) or the supraposture-first group (SF group; 10 subjects). Because the major limitation in most of the previous studies related to attention prioritization is the lack of specific instructions regarding to how the participants should direct their attention when performing dual tasks [14], we used a procedure derived from the "optimum-maximum method" proposed by Navon [15] to manipulate task priority in this study. The optimum-maximum method was used to guard the subjects' attention with specific instruction for both high-priority and low-priority tasks $[16,17]$. With this method, the high-priority task was designed as the "to-be-optimized" task, and the low-priority task was the "to-be-maximized" task. Participants were instructed to maintain an optimum performance level on the highpriority task and to perform their best on the low-priority task. Such a procedure requires participants to optimize the high-priority task and to not give up on the low-priority task. In addition, individually determined performance standard and feedback were provided in the

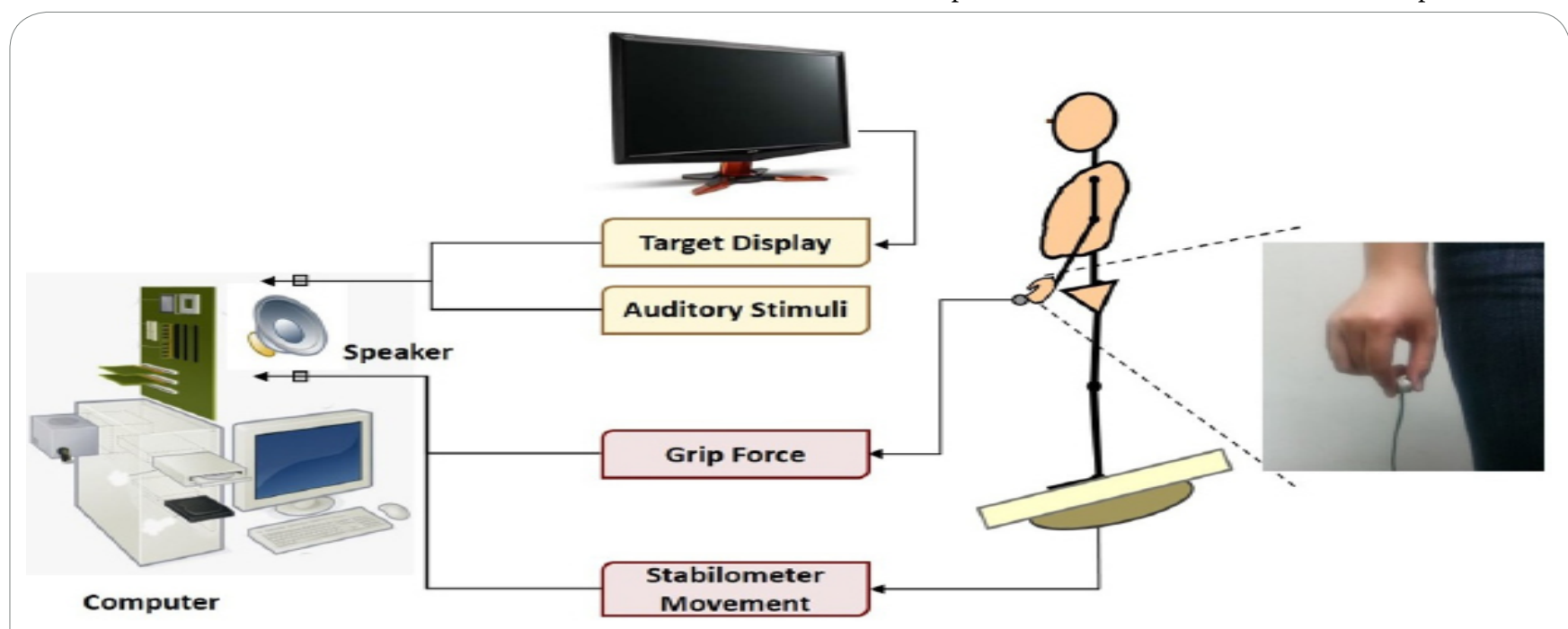

Figure 1: Diagram of experimental setup.

their balance on the stabilometer with an inclinometer (Model: FAS-A, MicroStrain, USA) mounted on the center of the stabilometer plate to measure the tilting angle of the stabilometer. The maximal anterior tilting angle was recorded before the experiment and 50\% of the maximal anterior tilting angle was set as the target angle for the postural task. For the suprapostural task, participants executed a thumb-index precision grip task, and the level of force output was recorded with a load cell $(15-\mathrm{mm}$ diameter $\times 10-\mathrm{mm}$ thickness, net weight $=7$ grams; Model: LCS, Nippon Tokushu Sokki Co., Japan). Maximum voluntary contraction (MVC) of the precision grip was recorded before the experiment and 50\% of the MVC was set as the target force of the suprapostural task. The participants needed to execute the thumb-index precision grip task in response to auditory cues. The auditory cues consisted of 80 -second sequences of tone pips, with a total of fifteen warning-executive signal pairs. The interval between a warning tone (frequency: $800 \mathrm{~Hz}$, duration: $100 \mathrm{~ms}$ ) and an executive tone (frequency: $500 \mathrm{~Hz}$, duration: $100 \mathrm{~ms}$ ) was 1.5 to-be-optimized task but not for to-be-maximized task. In this study, visual feedback about the target and performance of stabilometer movement and force-matching was used to enhance the attention priority. For example, participants in the PF group were instructed to concentrate more on the postural task, maintaining the tilting angle of the stabilometer at the target angle precisely and to maximize the precision of force-matching. Visual feedback of the stabilometer target angle and instantaneous stabilometer tilting angle were provided in the PF group, but visual information about the force target and force-output was not. In contrast, participants in the SF group were instructed to concentrate more on the precision grip task with coupling the force peak with the target precisely, and to maximize the precise tilting angle of the stabilometer. Visual feedback of force target and force-output of the load cell were provided in the SF group, but visual information about the stabilometer movement and its target angle was not. In addition, visual feedback for the target force for the $\mathrm{PF}$ group and the target tilting angle for the SF group were provided 
Citation: Yu SH, Huang CY (2015) Enhancing Dual-task Learning through Supraposture-first Instruction in Healthy Young Adults. Int J Phys Ther Rehab 1: 105. doi: http://dx.doi.org/10.15344/ijptr/2015/105

Page 3 of 5

as reminders. In each trial, we provided the visual feedback for the first three force-matching performances and the first 10 secondstabilometer tilting angle with their targets for the PF group and the SF group respectively. All the visual feedback was presented on a 22inch computer monitor $60 \mathrm{~cm}$ in front of subjects at eye-level. Besides the dual-task, the subjects needed to performed the corresponding single task condition for both postural and suprapostural tasks. units were converted to degrees. To assess the performance of the postural tasks and suprapostural tasks, we calculated the error and variance of both tasks.

\section{Data recording}

This study was conducted over a period of three successive days. The first day and the third day were pre-learning and post-learning phases respectively and the second day was the learning phase. There were five trials for the three experimental conditions (The dual-task task and its corresponding single posture and single force-matching precision grip conditions) in both pre-learning and post-learning tests. In the learning phase, participants performed the posturalsuprapostural dual-task for 10 trials with assigned task priority. A one-minute break was given after every trial to minimize fatigue. Both inclinometer and load cell data were digitized at a sample rate of $1 \mathrm{kHz}$.

\section{Data analyses}

The inclinometer data were conditioned with $6-\mathrm{Hz}$ low-pass filter and the units were converted to degrees. To assess the performance of the postural tasks and suprapostural tasks, we calculated the error and variance of both tasks. Postural error was presented by calculating the root mean square of the mismatch between the target angle and the stabilometer tilting angle. The approximate entrophy (ApEn) of the stabilometer tilting angle's trajectory was used to represent the variability property of the postural task. The value of the ApEn is between 0 and 2, with a value of closer to 2 representing higher irregularities, or larger complexity of the postural movement changes [18]. The force-matching error was presented as $\frac{|P G F-T F|}{T F} \times 100 \%$ (PGF: peak precision grip force, TF: target force), and the coefficient variation $(\mathrm{CV})$ of the peak precision grips was used to represent the variability property of the force control. To assess the effect on postural and suprapostural performance when executing posturalsuprapostural dual-tasks with a different task priority, the dual-task effect (DTE) [19] was calculated for each parameter. We calculated the DTE values for the task errors, CV of peak precision-grip force and ApEn of stabilometer movement as follows:

DTE $(\%)=[($ dual-task performance - single-task performance $) /$ single-task performance] $\times 100$

Positive DTE values indicate an increase in task error or task variance under dual-task conditions compared to single-task condition, representing more dual-task cost. In contrast, negative DTE values represent the dual-task benefit. The attention prioritization (PF, SF) and learning (pre-learning, post-learning) effects on posture and supraposture parameters, including the DTE values of postural error, force error, postural ApEn, and force $\mathrm{CV}$, were compared with repeated-measures analysis of variance (ANOVA). When necessary, post hoc least significant difference (LSD) comparisons were performed. The level of significance was set at $p=0.05$. Signal processing of behavioral data and statistical analysis were completed by using Matlab v. R2012a (Mathworks, Natick, MA, USA) and the statistical package for SPSS statistics v. 17.0 (SPSS Inc., Chicago, IL, USA).

\section{Results}

\section{Suprapostural task performance}

Figure 2 shows the DTE values of force error and force CV which represents the force-matching precision and variability for PF and SP groups during pre-learning and post-learning phases. For the forcematching error, significant attention prioritization $\left(\mathrm{F}_{1,9}=1.38 ; p<.01\right)$ and learning $\left(\mathrm{F}_{1,9}=16.92 ; p<.01\right)$ effects were observed. Post-hoc evaluation further revealed that the force-matching error was reduced after learning $(\mathrm{p}<.05)$ for both PF and SF groups (Figure 1, left). However, force-matching errors were greater in the PF group during pre-learning and post-learning phases than those in the SF group ( $p$ $<.05)$. We found a negative DTE values for the force-matching error $(-11.11 \pm 4.23 \%)$ of the SF group during the post-learning phase. This indicated that force-matching precision was better in the dualtask condition than that in the single force-matching condition while subjects focused on the precision grip task during dual-task learning. For the DTE values of force CV (Figure 2, right), both the PF and SF groups decreased the variability property of force control after learning (SF: pre-learning $=12.44 \pm 4.98 \%$, post-learning $=-5.21$ $\pm 4.66 \%, p<.05$; PF: pre-learning $=15.23 \pm 6.02 \%$, post-learning $=-6.47 \pm 4.94 \%, p<.05)$, but no significant difference was found between the two groups.
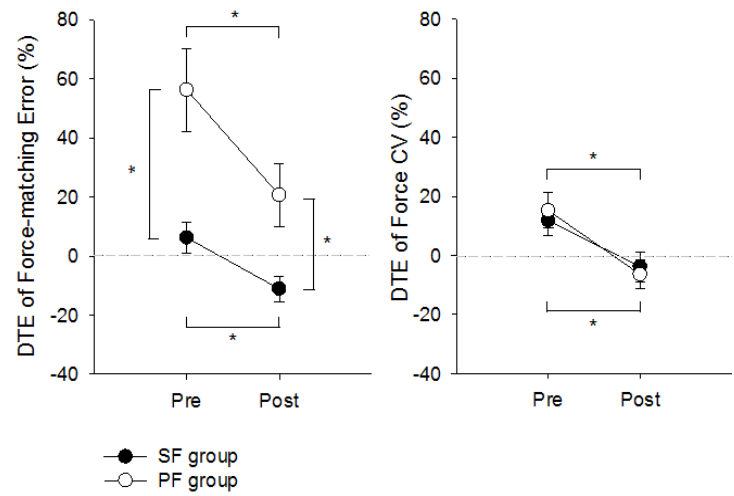

Figure 2: Mean and standard error of DTE values of forcematching error and force CV between the PF and SF groups during pre-learning and post-learning tests. (PF: posture-first; SF: supraposture-first; Pre: pre-learning test; Post: post-learning test).

\section{Postural task performance}

Figure 3 displays the means and standard error of DTE values of postural error and postural ApEn of SF and PF groups during prelearning and post-learning phases. The ANOVA suggested that the postural error varied with task priority $\left(\mathrm{F}_{1,9}=9.54 ; p<.05\right)$ and had a significant interaction between task priority and learning effects $\left(\mathrm{F}_{1,9}=11.15 ; p<.01\right)$. The postural error DTE values decreased after learning in the SF group (pre-learning $=1.81 \pm 4.60 \%$, post-learning $=-11.07 \pm 3.64 \%, p<.05$ ) but increased in the PF group (pre-learning $=1.73 \pm 1.84 \%$, post-learning $=10.28 \pm 2.66 \%, p<.05$ ) (Figure 2 , left). This result indicated that the dual-task cost of postural performance increased while learning with the posture-first strategy. The postural error was greater in the PF group than in the SF group during postlearning phase $(p<.01)$, a different that was not seen during the prelearning phase. 


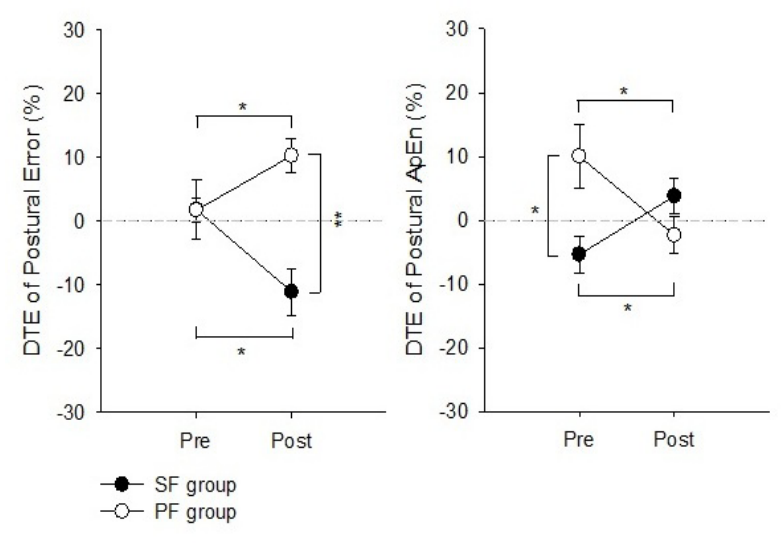

Figure 3: Mean and standard error of DTE values of postural error and postural ApEn between the PF and SF groups during pre-learning and post-learning tests. (PF: posture-first; SF: supraposture-first; Pre: pre-learning test; Post: post-learning test).

The ANOVA results showed that there was no priority and learning effects on postural ApEn, but showed a significant interaction between task priority and learning effect $\left(\mathrm{F}_{1,9}=14.20 ; p<.01\right)$. A post-hoc analysis revealed that the SF group had a lower DTE value of postural ApEn relative to the PF group before training $(p<.05)$ (Figure 2, right). However, after learning, postural ApEn decreased in the PF group (pre-learning: $10.02 \pm 4.98$; post-learning: $-2.34 \pm 2.84$, $p<.05$ ) but increased in the SF group (pre-learning: $-5.39 \pm 2.86 \%$, post-learning: $3.80 \pm 2.81 \%, p<.05$ ) indicating postural variance varied depending on different attention prioritization strategy.

\section{Discussion}

\section{A decrease in postural accuracy with posture-first learning}

This study provides evidence that a dual-task learning program with supraposture-first strategy is effective in improving postural and suprapostural accuracy. After the 1-day intervention, participants in the SF group significantly improved performance on accuracy of both force-matching and postural tasks. Even though the PF and SF groups were equally effective at improving force-matching performance, including reducing the DTE values of force-matching error and variability, the SF group was superior to the PF group in forcematching accuracy in both pre-learning and post-learning phases. The results are in line with previous studies $[9,19]$. During walking or narrow standing while performing an auditory cognitive task, the reaction time of the cognitive task was much shorter when focusing on the cognitive task than when focusing on posture. In addition, we found that the SF group reversed the DTE value of force-matching from positive to negative after learning. This finding suggests that with the supraposture-first strategy, the phenomenon of "dual-task cost" transfers to "dual-task benefit" for suprapostural performance even with a very short learning session.

Surprisingly, we found that dual-task learning with the posture-first strategy degraded postural accuracy. However, with the supraposturefirst strategy, postural accuracy improved. This result is in agreement with a study showing increased dual-task cost of postural accuracy while focusing on postural task when performing a stepping task (posture task) and an auditory Stroop task simultaneously [20].
Our results support the constrained action hypotheses which states that improvements in postural control should be apparent when prioritizing attention away from the posture itself, suggesting that increasing the level of controlled processing of posture will increase postural instability in young adults [21]. Furthermore, recent studies have demonstrated functional adjustments of postural sway in response to suprapostural task goals. For instance, postural sway was found to be reduced when the more attention was required for suprapostural tasks $[22,23]$. Thus, the motor system seems to be able to automatically adjust posture to the demands of other required tasks.

\section{An increase in postural irregularity with supraposture-first learning}

One of the important findings in this study is that dual-task learning by focusing on the force-matching task decreased postural regularity with increased postural ApEn. In contrast, learning by focusing on the stabilometer movement increased postural regularity with decreased postural ApEn (Figure 3). The ApEn has been used to characterize stochastic features of postural performance while standing on a force plate or on a stabilometer [24-26]. A more regular CoP signal or stabilometer movement is associated with increased attentional investments in postural control, reflecting less automaticity or postural control. For pathological groups such as patients with stroke or ligament laxity, postural sway is more regular (low value of ApEn) than for healthy controls during quiet standing $[24,25]$. According to Roerdink et al., CoP trajectories are more regular in stroke patients than in controls and become less regular when performing a secondary cognitive task while standing [24]. This suggests that the measure of complexity or irregularity of a system is linked to efficiency or automaticity of postural control. All activities that are over-learned would execute automatically using minimum attentional resources and do not stress the capacity limitations of the system or demand excessive attention [27]. According to our ApEn results, participants who learned the dual task using prioritizing posture concentrated more on postural task and interrupted the automatized postural control, resulting in greater postural errors. Moreover, increased postural regularity in the PF group may have been caused by participants adopting a more rigid posture with a posture-first strategy [11]. These findings also provide support for the idea that focusing attention away from posture allows for "functional variability" [28], such that the motor systems automatically adjust the various degrees of freedom to achieve postural balance and facilitate force-matching control.

\section{Study Limitations}

Our results provide little support for a theoretical position that frames the attention prioritization interaction between postural and suprapostural control. Rather, this study supports the proposition that the supraposture-first strategy is more efficient for dual-task learning in young adults than the posture-first strategy. However, it is conceivable that the dual-tasking effects are modulated by individual differences in attentional capacity. With increasing age, the efficacy of sensory and muscular systems involved in postural control are reduced resulting in more limited attentional capacity for performing dual tasks [29]. Indeed, lack of flexibility when focusing attention between postural and suprapostural tasks was observed in older adults [30]. Therefore, the supraposture-first strategy may not be the most appropriate action strategy in dual-task learning for older adults who cannot manage the attentional cost of both tasks. Future studies are needed to identify the attention prioritization effects on dual-task training in older adults for achieving optimized dual-task 
Citation: Yu SH, Huang CY (2015) Enhancing Dual-task Learning through Supraposture-first Instruction in Healthy Young Adults. Int J Phys Ther Rehab 1: 105. doi: http://dx.doi.org/10.15344/ijptr/2015/105

Page 5 of 5

performance and minimizing the risk of falling under dual-task conditions.

\section{Conclusion}

Attention prioritization is important for improving dualtask performances. More specifically, dual-task learning with supraposture-first strategy can enhance suprapostural and postural accuracy, and increase functional variability of posture to optimize postural-suprapostural control. Dual-task learning under posturefirst instruction may not benefit postural balance due to disturbing automatic processing of postural control. These findings may help in training dual-task performances in healthy young and older people in both the clinical and research settings.

\section{Competing Interests}

The authors have declared that no competing interests exist.

\section{Author Contributions}

Shu-Han Yu: acquisition of data, analysis of data and drafting the manuscript.

Cheng-Ya Huang: study conception and design, interpretation of data, drafting and revising manuscript.

\section{Funding}

This research was supported by a grant from the National Science Council, R.O.C. Taiwan, under grant no. NSC 102-2314-B-002-014 and partially supported by a grant from the Ministry of Science and Technology, Taiwan, under grant no. MOST 103-2314-B-002-007MY3.

\section{References}

1. Stoffregen TA, Smart LJ, Bardy BG, Pagulayan RJ (1999) Postura stabilization of looking. J Exp Psychol Hum Percept Perform 25: 1641 1658.

2. Woollacott M, Shumway-Cook A (2002) Attention and the control of posture and gait: a review of an emerging area of research. Gait Posture 16: 1-14.

3. Cluff T, Gharib T, Balasubramaniam R (2010) Attentional influences on the performance of secondary physical tasks during posture control. Exp Brain Res 203: 647-658.

4. Tombu M, Jolicoeur P (2003) A central capacity sharing model of dual-task performance. J Exp Psychol Hum Percept Perform 29: 3-18.

5. Bloem BR, Valkenburg VV, Slabbekoorn M, Willemsen MD (2001) The multiple tasks test: Development and normal strategies. Gait Posture 14: 191-202.

6. Yogev-Seligmann G, Rotem-Galili Y, Mirelman A, Dickstein R, Giladi N et al. (2010) How does explicit prioritization alter walking during dual-task performance? Effects of age and sex on gait speed and variability. Phys Ther 90: 177-186.

7. Bloem BR, Valkenburg VV, Slabbekoorn M, van Dijk JG (2001) The multiple tasks test. Strategies in Parkinson's disease. Exp Brain Res 137: 478-486.

8. Bloem BR, Grimbergen YA, van Dijk JG, Munneke M (2006) The "posture second" strategy: a review of wrong priorities in Parkinson's disease. J Neurol Sci 248: 196-204

9. Kelly VE, Eusterbrock AJ, Shumway-Cook A (2013) Factors influencing dynamic prioritization during dual-task walking in healthy young adults. Gait Posture 37: 131-134.

10. Siu K-C, Siu M, Woollacott (2007) Attentional demands of postural control The ability to selectively allocate information-processing resources. Gait Posture 25: 121-126.

11. Shumway-Cook A, Woollacott M, Kerns KA, Baldwin M (1997) The effects of two types of cognitive tasks on postural stability in older adults with and without a history of falls. J Gerontol A Biol Sci Med Sci 52: M232-240.
12. Silsupadol P, Siu KC, Shumway-Cook A, Woollacott MH (2006) Training of balance under single- and dual-task conditions in older adults with balance impairment. Phys Ther 86: 269-281.

13. Silsupadol P, Lugade V, Shumway-Cook A, van Donkelaar P, Chou LS, et al. (2009) Training-related changes in dual-task walking performance of elderly persons with balance impairment: a double-blind, randomized controlled trial. Gait Posture 29: 634-639.

14. Siu KC, Woollacott MH (2007) Attention demands of postural control: the ability to selectively allocate information-processing resources. Gait Posture 25: 121-126.

15. Navon D (1990) Exploring two methods for estimating performance tradeoff. Bull Psychon Soc 28: 155-157.

16. Zanone PG, Monno A, Temprado JJ, Laurent M (2001) Shared dynamics of attentional cost and pattern stability. Hum Mov Sci 20: 765-789.

17. Tsang PS (2013) Ageing and attentional control. Q J Exp Psychol (Hove) 66: 1517-1547.

18. Donker SF, Roerdink M, Greven A, Beek PJ (2007) Regularity of centerof-pressure trajectories depends on the amount of attention invested in postural control. Exp Brain Res 181: 1-11.

19. Remaud A, Boyas S, Lajoie Y, Bilodeau M (2013) Attentional focus influences postural control and reaction time performances only during challenging dual-task conditions in healthy young adults. Exp Brain Res 231: $219-229$.

20. Liston MB, Bergmann JH, Keating N, Green DA, Pavlou M (2014) Postural prioritization is differentially altered in healthy older compared to younger adults during visual and auditory coded spatial multitasking. Gait Posture 39: 198-204.

21. Wulf G, Prinz W (2001) Directing attention to movement effects enhances learning: a review. Psychon Bull Rev 8: 648-660.

22. Huang CY, Cherng R-J, Hwang I-S (2010) Reciprocal influences on performances of a postural-suprapostural task by manipulating the level of task-load. J Electromyogr Kinesiol 20: 413-419.

23. Jehu DA, Desponts A, Paquet N, Lajoie $Y$ (2015) Prioritizing attention on a reaction time task improves postural control and reaction time. Int $\mathrm{J}$ Neurosci 125: 100-106.

24. Roerdink M, De Haart M, Daffertshofer A, Donker SF, Geurts AC, et al (2006) Dynamical structure of center-of-pressure trajectories in patients recovering from stroke. Exp Brain Res 174: 256-269.

25. Rigoldi C, Cimolin V, Camerota F, Celletti C, Albertini G, et al. (2013) Measuring regularity of human postural sway using approximate entropy and sample entropy in patients with Ehlers-Danlos syndrome hypermobility type. Res Dev Disabil 34: 840-846.

26. Huang CY, Zhao CG, Hwang IS (2014) Neural basis of postural focus effect on concurrent postural and motor tasks: phase-locked electroencephalogram responses. Behav Brain Res 274: 95-107.

27. Schneider W, Shiffrin RM (1977) Controlled and automatic human information processing: I. Detection, search, and attention. Psychol Rev 84: 1-66.

28. Wulf G (2013) Attentional focus and motor learning: A review of 15 years. Int Rev Sport Exerc Psychol 6: 77-104.

29. Huxhold O, Li SC, Schmiedek F, Lindenberger U (2006) Dual-tasking postural control: Aging and the effects of cognitive demand in conjunction with focus of attention. Brain Res Bull 69: 294-305

30. Borel L, Alescio-Lautier B2 (2014) Posture and cognition in the elderly: interaction and contribution to the rehabilitation strategies. Neurophysiol Clin 44: 95-107. 